\title{
Implementing the conjugate gradient algorithm on multi-core systems
}

\author{
W.A. Wiggers and V. Bakker and A.B.J. Kokkeler and G.J.M. Smit \\ University of Twente, Department of EEMCS \\ P.O. Box 217, 7500 AE Enschede, The Netherlands
}

\begin{abstract}
In linear solvers, like the conjugate gradient algorithm, sparse-matrix vector multiplication is an important kernel. Due to the sparseness of the matrices, the solver runs relatively slow. For digital optical tomography (DOT), a large set of linear equations have to be solved which currently takes in the order of hours on desktop computers. Our goal was to speed up the conjugate gradient solver. In this paper we present the results of applying multiple optimization techniques and exploiting multi-core solutions offered by two recently introduced architectures: Intel's Woodcrest general purpose processor and NVIDIA's G80 graphical processing unit. Using these techniques for these architectures, a speedup of a factor three has been achieved.
\end{abstract}

\section{INTRODUCTION}

In linear solvers, like the conjugate gradient (CG) algorithm [1], sparse-matrix vector multiplication (SMVM) is an important kernel. Due to the sparseness of the matrix and the lack of temporal and spatial locality, the SMVM kernel runs inefficiently on general purpose processors. Fast execution of the multiplication is needed, since the linear solvers require multiple iterations.

SMVM offers parallelism, which can be exploited using parallel computing. Currently there is a trend in the semiconductor industry to move from single-core designs in General Purpose Processors (GPPs) to multi-core designs. Another trend is using a Graphics Processing Unit (GPU) for general purpose programming [2]. Both architectures offer a lot of processing power employing multiple processing units which can be used in parallel. This paper shows the results of running a CG algorithm on multi-core systems.

We have implemented a conjugate gradient solver using two Intel 5140 Woodcrest GPPs and using NVIDIA's G80 GPU. First, in Section II the necesarry information about the used architectures is presented. In Section III techniques for improving the performance are explained. Results are presented in Section IV. This paper is finalized with the conclusions in Section V.

\section{ARChitectures}

The increase in performance of single-core processors is slowing down because of limited growth in clock frequencies [3] and limited architectural improvements for superscalar designs [4]. This has caused a trend towards multi-core designs.

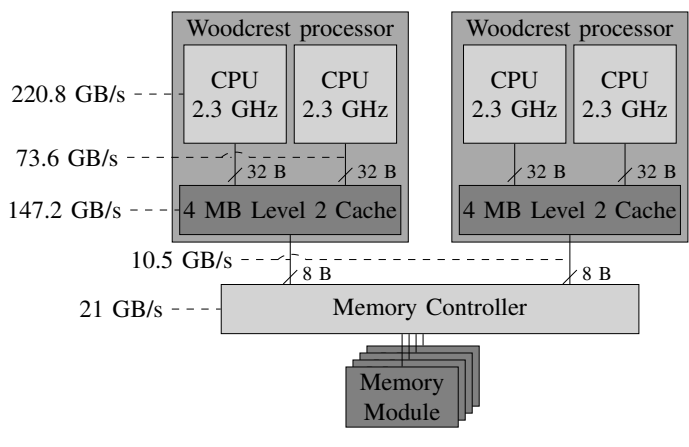

Fig. 1. Bensley architecture memory model

\section{A. Intel Xeon 5140 Woodcrest}

In addition to further optimizing single-core processors, Intel chose to place multiple processors on a single chip. The Woodcrest processor contains two cores based on Intel's Core micro architecture. In Intel's Roadmap, quad-core processors are announced and octa-core processors are the next logical step. The memory model of the Bensley architecture, including two Woodcrest processors, is depicted in Figure 1.

The processing cores run at $2.3 \mathrm{GHz}$. In the ideal case, in each cycle 6 SSE instructions are executed in parallel at each core. Each SSE instruction requires 16 bytes of data which results in a total data bandwidth of $220.8 \mathrm{~GB} / \mathrm{s}$ to fully utilize a processing core (assuming the program fits in cache). Each core has $16 \mathrm{~KB}$ Level 1 data cache and $16 \mathrm{~KB}$ Level 1 instruction cache. The Level 2 cache connected with two separate 256-bit wide buses also runs at $2.3 \mathrm{GHz}$, delivering $147.2 \mathrm{~GB} / \mathrm{s}$.

As depicted in the Figure 1, two cores share a total of $4 \mathrm{MB}$ of Level 2 cache. The Woodcrest architecture reduces memory access latency by using eight hardware prefetchers. In case an application has limited spatial and temporal locality (such as SMVM), the memory hierarchy does not offer sufficient bandwidth to constantly provide the processing cores with data, causing the cores to stall.

\section{B. NVIDIA GeForce 8800 GTX}

The GeForce 8800 GTX architecture, as depicted in Figure 2, uses the G80 processor which has 128 stream processors running at $1.35 \mathrm{GHz}$ while the rest of the processor runs at $675 \mathrm{MHz}$. There is a 384-bit wide bus to the main memory. The 768 MB main memory uses Graphics Double Data Rate 3 
memory running on $1.8 \mathrm{GHz}$. This leads to a peak memory bandwidth of $86.4 \mathrm{~GB} / \mathrm{s}$.

Eight stream processing cores are grouped into one multiprocessor and share a $16 \mathrm{~KB}$ memory. Each stream processor can process a 32-bit floating point number calculation every cycle at a frequency of $1.35 \mathrm{GHz}$. This results in a total data processing capacity of $43.2 \mathrm{~GB} / \mathrm{s}$. The 16 multiprocessors are connected to main memory via a 48 byte bus clocked at $1.8 \mathrm{GHz}$. The GPU offers a lot of processing power compared to a GPP, caused by dedicating more raw transistors to calculations than the GPP.

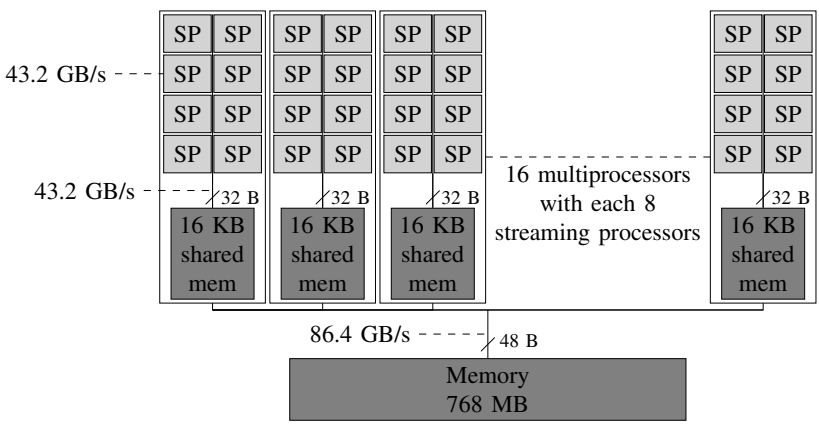

Fig. 2. GeForce architecture memory model

Traditional GPU programming differs from programming a GPP since GPUs are initially designed for 3D rendering. NVIDIA introduced a programming model, called CUDA (Compute Unified Driver Architecture). Within CUDA the GPU is viewed as co-processor to which data-intensive and highly parallel tasks can be off-loaded. CUDA provides libraries with common mathematical functions. The GPU can be programmed using (extended) $\mathrm{C}$, without knowledge about conventional GPU pipelines.

\section{Algorithm TEChNiQues}

Different architectures call for different optimization techniques to maximize throughput. For both architectures, the chosen optimization techniques are shortly discussed.

\section{A. Intel Xeon 5140 Woodcrest}

Three main factors limit the performance of sparse-matrix multiplications on modern superscalar processors [5]. First, data structures that represent sparse-matrices have no temporal locality (the matrix elements are used only once) and limited spatial locality (data accesses are in a stride loop). Second, the tendency of multiple load/store function units to miss on the same cache line, as a consequence of the data access pattern of the loop. Finally, sparse matrix vector multiplication codes typically perform a large number of load instructions relative to the number of floating-point instructions. Since the memory is not fast enough to offer new data, the floating-point units are underutilized.

The sparse-matrix elements are usually reordered in such a way that most non-zero elements will be close to the diagonal of the matrix. This improves memory access during the multiplication of the matrix, since spatial locality is improved.

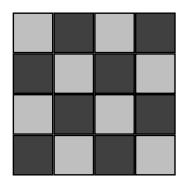

(a) Even odd

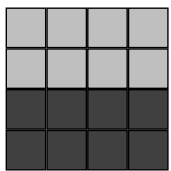

(b) Block row

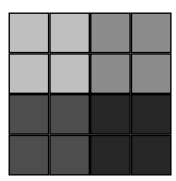

(c) Submatrix
Fig. 3. Multiple ways to split up a $4 \times 4$ matrix

As summarized in [5], multiple reordering algorithms exist. In this paper, the Cuthill-McKee (CM) algorithm is used. The Cuthill McKee [6] heuristic algorithm tries to minimize the bandwidth of the matrix. The bandwidth $B$ of a matrix $A$ is defined as $B(A)=1+\max (|i-j|)_{A i, j \neq 0}$.

By storing the sparse-matrix in the Compressed Row Format (CRF) [7], only the non-zero elements are stored. CRF uses three arrays; the first array is used to store all non-zero elements. The second array stores all column indexes of the non-zero elements. The last array stores the locations in the arrays that start a row. Using the CRF leads to a smaller data set and better spatial locality. The dataset is reduced from $n^{2}$ elements to $2 \times n n z+n+1$ ( $n n z=$ number of non-zeros). Our test suite consisted of a matrix of $138,324 \times 138,324$ $(n=138,324)$ elements, containing around $2.5 \times 10^{6}$ nonzero elements.

By using Intel's Math Kernel Library (MKL) for our CG solver specific hardware features such as SSE instructions, loop unrolling etc. are exploited. We used the OpenMP library for implementing data parallelism for our dual-core processors. We have experimented with multiple ways to parallelize the SMVMs by splitting up the matrix.

When using even odd splitting (see Figure 3(a)), matrix elements which are direct neighbors are split up in two separate sets. The data sets are symmetric along the matrix diagonal. When the results of two sets are combined, bigger numerical errors occur since the results of different sets are not kept in extra wide SSE accumulator registers. In effect, the amount of CG iterations needed to converge increases. We observed a doubling in the number of iterations thus making this method practically useless.

Another way to balance the load can be seen in Figure 3(b). The matrix is split up into an upper and lower part. A row always fits completely within one of the two sets. The disadvantage is that we need the double amount of memory to store the system matrix since symmetry is lost. We have measured that this method needs $\approx 30 \%$ more clock cycles on the Woodcrest processor than the method we describe next.

By splitting the matrix in small square submatrices (see Figure 3(c)), we can retain the symmetry within the diagonal submatrices. This method does not have numerical errors like the even-odd splitting. Further scaling to more-core processors is also possible since we can repeat the same splitting for the diagonal submatrices. For these reasons we use the submatrix splitting method for implementing data parallelism within the CG algorithm on the Woodcrest architecture.

Our DOT algorithm requires multiple linear equations to be 
solved. Multiple threads are used to parallelize the execution of multiple solvers over the two processors. Each CG solver requires multiple SMVMs. The SMVM is parallelized over the two cores of one processor using the submatrix splitting technique.

\section{B. NVIDIA GeForce 8800 GTX}

To obtain a reasonable execution time on the GeForce it is important that the 300 cycle memory latency is hidden when fetching data from memory. To hide this latency we require that there are multiple arithmetic operations per data fetch. Furthermore, the available streaming processors and multiprocessors are loaded with more threads than can actually run on the device such that the GPU's thread processor can switch between threads that stall on memory fetches. By hiding memory access latency we allow for a more efficient use of available resources and thus lower the total execution time. This is also explained in [8] which describes the Parallel Random Access Model (PRAM) model for programming architectures with a large number of processors. The programming paradigm used by NVIDIA's CUDA C-compiler is based on this model. We used this PRAM model as well to design the custom kernel needed to implement the sparse matrix vector multiplication.

To execute a SMVM, it is inefficient to distribute parts of a row of the matrix over multiple processors. Each processor can only calculate a part of the "row-vector" product and the final combination stage will introduce additional interprocessor communication overhead. We chose to let every thread work on a row of the matrix. This streaming method is also described in [9].

Using CRF introduces memory redirections, since the locations of all elements in the matrix have to be calculated. By padding every column of the matrix with zeros such that every row has an equal number of elements, the number of memory redirections is reduced. Since every row has the same amount of elements, we can calculate the index of the row.

Every streaming processor needs to perform the same operation every clock cycle, thus we reordered the matrix in such a way that starting from the first row until the last row of the matrix, the number of elements increases. Consequently subsequent rows will have approximately the same number of non-zeros. We can use this to implement a branch when a zero element, resulting from padding, is loaded from memory since threads close to each other take the same branch. Generally the implementation of branches should be taken with great caution on the GPU. If two thread processors take a different branch the execution path needs to be serialized which is very resource inefficient. By reordering the matrix in the way described above this disadvantage is minimized.

\section{REsults}

In this section we will show results of our CG solver for DOT. Our initial solver was running on an Intel Xeon Cranford processor and is used as reference. The CG solver available on the Cranford system was implemented using the deal.II library (version 5.2.0), which was compiled with all optimizations using the Intel Compiler 8.1. For the Woodcrest architecture we used the Intel MKL library version 9.0. All results are measured with VTune 8.01. The Cranford PC is a single Intel Xeon Cranford $3.2 \mathrm{GHz}$ with $1 \mathrm{MB}$ of level 2 cache and $1 \mathrm{~GB}$ main memory. For the Woodcrest architecture we used two Intel Xeon 5140 Woodcrest $2.3 \mathrm{Ghz}$ processors with each a shared 4 MB level 2 cache (see Figure 1). The system has 2 GB memory divided over two channels on a Bensley platform. Both Intel processors offer 64-bit precision.

For the GPU measurements the NVIDIA GeForce 8800 GTX board was placed in the Woodcrest PC. The GeForce board was connected via a 16 lanes PCI-express bus. All timing was performed with the timing function provided by the CUDA environment. The GPU offers 32-bit precision. The precision influences the convergence rate, since numerical errors lead to slower convergence.

\section{A. Intel Xeon 5140 Woodcrest}

The results of the conjugate gradient solver on the Woodcrest is presented in Table I. The last column in the table shows the speedup compared to previously tested architecture and the initially used architecture respectively.

\begin{tabular}{|l|r|r|rr|}
\hline Processor / Implementation & $\begin{array}{r}\text { \#Iterations } \\
\text { per solve }\end{array}$ & Time(s) & \multicolumn{2}{|c|}{ Speedup } \\
\hline \hline Cranford / Deal.II & 152 & 3.07 & 1.0 & 1.0 \\
\hline Single-core Woodcrest / deal.II & 152 & 2.89 & 1.06 & 1.06 \\
\hline Single-core Woodcrest / MKL & 154 & 2.40 & 1.20 & 1.28 \\
\hline Dual-core Woodcrest / MKL & 154 & 1.59 & 1.51 & 1.93 \\
\hline
\end{tabular}

TABLE I

RESULTS OF THE CONJUGATE GRADIENT SOLVER ON THE WOODCREST

Although Intel's hand-optimized MKL library requires slightly more iterations to achieve equal precision than the initial compiler optimized deal.II implementation, a speed-up of a factor 1.20 is obtained. Analysis with VTune showed that $85 \%$ of all the required clock cycles of the CG solver are used for sparse-matrix multiplication.

A single single-core Woodcrest using deal.II is 1.06 times faster than the Cranford using deal.II, which is probably caused by the bigger cache size and improvements of the Core micro-architecture processor. The hand-optimized MKL clearly better exploits the processors hardware, since a speedup factor of 1.20 is achieved. Enabling the second core added an improvement of a factor 1.51. However, analysis with VTune showed that cache-misses and synchronization caused by the OpenMP library are becoming more important when adding more cores/processors.

Additional memory modules are used when using two dualcore Woodcrest processors to run multiple solvers in parallel. This increases the memory bandwidth to support the offered $21 \mathrm{~GB} / \mathrm{s}$ total memory bandwidth capacity. Experiments with multiple memory modules were done to see the effect of scaling memory bandwidth. Results are shown in Table II. The speed-up is relative to the execution time of a single dual-core processor. 


\begin{tabular}{|l|r|r|}
\hline Memory modules & Average per solve (s) & Speed-up \\
\hline \hline 1 & 1.49 & 1.07 \\
\hline 2 & 1.25 & 1.27 \\
\hline 4 & 1.00 & 1.59 \\
\hline
\end{tabular}

TABLE II

MULTI-PROCESSOR SPEED-UP FOR DIFFERENT NUMBER OF MEMORY

MODULES

For the dual-socket Woodcrest architecture implementation the speedup factor is not a factor 2 compared to the singlesocket Woodcrest architecture, but a factor 1.59. One reason for this is that logically separated solvers share physical memory modules causing memory bank conflicts.

\section{B. NVIDIA GeForce 8800 GTX}

In Table III the results of different SMVM implementations on the GeForce architecture are shown. The first row shows the execution time needed for the sparse matrix vector multiplication implementation described in the previous section. We have also simulated an approach where every thread loads the matrix values and indices in the $16 \mathrm{kB}$ shared memory and multiplies this with 32 different vectors. The execution time of this simulated approach is given in the second row.

\begin{tabular}{|l|r|}
\hline Function & Time $(\mathrm{ms})$ \\
\hline \hline SMVM & 3.40 \\
\hline SMVM (multiple vectors) & 1.22 \\
\hline
\end{tabular}

TABLE III

SPARSE MATRIX VECTOR MULTIPLICATION RESULTS ON THE GEFORCE

In Table IV, the results of the conjugate gradient solver on the GeForce architecture are shown. On average, around $4 \mathrm{~ms}$ is spent per iteration of the $\mathrm{CG}$ algorithm. However, due to the 32-bit precision, more iterations are required to converge compared to a 64-bit implementation.

\section{Precision}

The results of solvers have been compared to a MatLab solution. Although the GeForce is faster, the relative error of the solution is bigger than of the 64-bit GPP implementation. Since the MKL doesn't support 32-bit operations, we changed the Woodcrest implementation to allow a bigger residual (and thus relative error). When the relative errors of the Woodcrest and the GeForce are approximately equal, the execution time of the two architecture are almost the same.

\section{CONCLUSION}

The execution time of the CG algorithm on the GeForce and Woodcrest architectures is limited by the mismatch between

\begin{tabular}{|l|r|r|}
\hline Processor & \#Iterations per solve & Time(s) \\
\hline \hline NVIDIA GeForce 8800 GFX & 177 & 0.71 \\
\hline \multicolumn{2}{|l|}{} \\
\hline \multicolumn{2}{|l}{ TABLE IV }
\end{tabular}

TABLE IV

CONJugAte GRAdient SOlver Results ON THE GeForCE the bandwidth of the memory and the required memory bandwidth for the processing (also called the memory wall).

On the Woodcrest architecture this results in stalling due to level 2 cache misses. Together with load balancing of the sparse matrix on the cores, these stalling problems will be the performance limiting factors in the future.

On the GeForce architecture we see a similar memory wall. Memory access patterns on the GeForce architecture determine the obtained memory bandwidth and for the SMVM algorithm this is the main performance limiting factor. Since the GeForce architecture has software controlled caches it is possible to relief the pressure on the memory bandwidth. Unfortunately this requires a change in the original $\mathrm{CG}$ algorithm, which has not been implemented.

The Cranford processor needs an average of 3.07 seconds, a single Woodcrest (containing two cores) 1.59 seconds and the GeForce 0.71 seconds per solve. After applying all optimization techniques, using two Woodcrest architectures and multiple memory modules, a total speedup of a factor 3.07 on a GPP has been achieved.

The GeForce architecture has the lowest execution time per solve. Per iteration of the CG algorithm the GeForce is even a factor 2.56 faster than a single dual-core Woodcrest processor. This makes the GPU a good candidate for CG solvers.

However, the relative error of the results has to be taken into account to make a fair comparison between the 64-bit floating point and 32-bit floating point precision architectures. For results with an equal approximated relative error, the Woodcrest and GeForce architectures have approximately the same execution time.

\section{REFERENCES}

[1] J. R. Shewchuk, "An introduction to the conjugate gradient method without the agonizing pain," Pittsburgh, PA, USA, Tech. Rep., 1994.

[2] J. D. Owens, D. Luebke, N. Govindaraju, M. Harris, J. Krger, A. E. Lefohn, and T. J. Purcell, "A survey of general-purpose computation on graphics hardware," in Eurographics 2005, State of the Art Reports, Aug. 2005, pp. 21-51.

[3] D. Patterson, K. A. Arvind, D. Chiou, J. Hoe, C. Kozyrakis, S. Lu, M. Oskin, J. Rabaey, and J. Wawrzynek, "RAMP: Research Accelerator for Multiple Processors," Technical Record of the 18th Hot Chips Symposium, Palo Alto, CA, August 2006.

[4] J. L. Hennessy and D. A. Patterson, Computer Architecture, A Quantitative Approach. Morgan Kaufmann Publischers, 2003.

[5] S. Toledo, "Improving the memory-system performance of sparsematrix vector multiplication," IBM Journal of Research and Development, vol. 41, no. 6, pp. 711-725, 1997. [Online]. Available: citeseer.ist.psu.edu/toledo97improving.html

[6] E. Cuthill and J. McKee, "Reducing the bandwidth of sparse symmetric matrices," Proceedings of the 1969 24th national conference, pp. 157172, 1969.

[7] J. Dongarra, "Compressed Row Storage," in Templates for the Solution of Algebraic Eigenvalue Problems: A Practical Guide, Z. Bai, J. Demmel, J. Dongarra, A. Ruhe, and H. van der Vorst, Eds. Philadelphia: SIAM, 2000.

[8] S. Chatterjee and J. Prins, "Parallel and Distributed computing PRAM Algorithms," www.cs.unc.edu/prins/Classes/203/Handouts/pram.pdf, 2005, accessed 27-jan-2007, University of North Carolina.

[9] J. Gummaraju and M. Rosenblum, "Stream Programming on GeneralPurpose Processors," in MICRO 38: Proceedings of the 38th annual ACM/IEEE international symposium on Microarchitecture, 2005. 